\title{
ARTICLE OPEN Effective mass and Fermi surface complexity factor from ab initio band structure calculations
}

\author{
Zachary M. Gibbs ${ }^{1}$, Francesco Ricci ${ }^{2}$, Guodong Li ${ }^{3}$, Hong Zhu ${ }^{4}$, Kristin Persson ${ }^{5}$, Gerbrand Ceder ${ }^{4,5}$, Geoffroy Hautier ${ }^{2}$, \\ Anubhav Jain ${ }^{5}$ and G. Jeffrey Snyder (iD $)^{3}$
}

The effective mass is a convenient descriptor of the electronic band structure used to characterize the density of states and electron transport based on a free electron model. While effective mass is an excellent first-order descriptor in real systems, the exact value can have several definitions, each of which describe a different aspect of electron transport. Here we use Boltzmann transport calculations applied to ab initio band structures to extract a density-of-states effective mass from the Seebeck Coefficient and an inertial mass from the electrical conductivity to characterize the band structure irrespective of the exact scattering mechanism. We identify a Fermi Surface Complexity Factor: $N_{v}^{*} K^{*}$ from the ratio of these two masses, which in simple cases depends on the number of Fermi surface pockets $\left(N_{\mathrm{v}}^{*}\right)$ and their anisotropy $K^{*}$, both of which are beneficial to high thermoelectric performance as exemplified by the high values found in PbTe. The Fermi Surface Complexity factor can be used in high-throughput search of promising thermoelectric materials.

npj Computational Materials (2017)3:8; doi:10.1038/s41524-017-0013-3

\section{INTRODUCTION}

The calculation of electronic band structures using density functional theory (DFT) is now so routine that it is becoming faster to compute certain physical properties than make samples and measure them-inspiring the materials genome initiative efforts worldwide. Ab initio calculations are important from a materials' design perspective in that they provide insight into the underlying electronic states that give rise to experimentally measurable properties. Dielectric, optical and transport properties such as electrical conductivity, Hall effect, and thermoelectric power (Seebeck effect) require knowledge not only of the electronic structure readily available from ab initio calculations, but may also require an assumption about the scattering. Using a constant relaxation time approximation, very precise predictions of transport properties that depend on fine details of the band structure can be made, however, the electrical conductivity predicted for instance can be greatly misleading because the relaxation time is approximated, often to an arbitrary constant. A recent study performed by the authors demonstrated that while Seebeck coefficient was reproduced fairly well across a variety of compounds (provided that the band gap was not severely underestimated), the experimental values on conductivities can be highly inaccurate using a constant relaxation time. ${ }^{1}$ While some scattering mechanisms can now be calculated using ab initio methods, they are far from routine and require special algorithms. The goal of this study is to extract transport information from band structure calculations that does not depend on any scattering assumption.

In the common free-electron approximation lexicon of electronic and optical properties we typically describe charge carriers as having an effective mass $m^{*}$ and a relaxation or scattering time $\tau$. Combining with the density of carriers $n$, the electrical conductivity $\sigma=n e^{2} \tau / m^{*}$ and drift mobility $\mu_{\mathrm{d}}=e \tau / m^{*}$ can also be expressed. This description, while not exact, has proven immensely helpful in the understanding and engineering of electronic materials that have profoundly changed our civilization. This representation already separates transport into electronic structure (through the $m^{*}$ ) and scattering $(\tau)$ terms as well as allowing the flexibility of varying $n$ (through doping, for example). It is, therefore, natural to expect that knowledge of electronic structure should reveal the appropriate effective mass for various values of $n$ but not necessarily the scattering-dependent transport properties (such as $\sigma$ and $\mu_{\mathrm{d}}$ ) until the scattering is known.

The free-electron description has been integral to semiconductor physics despite many examples of profound deviations. In the free-electron model the electronic structure is represented by a single, isotropic Fermi surface described by particle with mass and charge of an electron. A free-electron like description is only helpful to describe real semiconductors if we allow multiple $\left(N_{\mathrm{v}}\right)$ free-electron like pockets to describe the Fermi surface and that each pocket may be anisotropic (described by anisotropy term $K$ ) with effective mass $m^{*}$ that differs from free-electron mass. Because of the multiple pockets the total density of electronic states will be $N_{\mathrm{v}}$ times that of a single pocket.

In thermoelectrics, for example, these and other material parameters are helpful to predict the promise of a material for use in a thermoelectric device. ${ }^{2-4}$ For typical semiconductor transport where the electrons are scattered by acoustic phonons (deformation potential scattering) $^{5}$ the thermoelectric quality factor $B$, given by:

$$
B=\frac{2 k_{\mathrm{B}}^{2} \hbar}{3 \pi} \frac{N_{\mathrm{v}}}{m_{\mathrm{c}}^{*}} \frac{C_{l}}{\kappa_{\mathrm{L}} \bar{\Xi}^{2}} T
$$

\footnotetext{
${ }^{1}$ California Institute of Technology, Division of Chemistry and Chemical Engineering, Pasadena, CA, USA; ${ }^{2}$ Institute of Condensed Matter and Nanosciences (IMCN), Université catholique de Louvain, Chemin des étoiles 8, bte L7.03.01, Louvain-la-Neuve, Belgium; ${ }^{3}$ Department of Materials Science and Engineering, Northwestern University, 2220 Campus

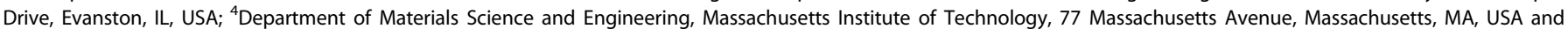
${ }^{5}$ Lawrence Berkeley National Lab, 1 Cyclotron Road, Berkeley, CA, USA Correspondence: G Jeffrey Snyder (jeff.snyder@northwestern.edu)
}

Received: 30 October 2016 Revised: 13 January 2017 Accepted: 25 January 2017 Published online: 23 February 2017 
determines the maximum $z T$ (materials efficiency) when the semiconductor is optimally doped, where $\kappa_{\mathrm{L}}$ is the lattice thermal conductivity, $\equiv$ is the deformation potential, $C_{I}$ is the average longitudinal elastic modulus, $m_{c}^{*}$ is the conductivity effective mass, and $T$ is temperature.

Two materials parameters, valley degeneracy $N_{v}$ and conductivity effective mass $m_{c}^{*}$ appear in the quality factor that concern the static band structure and should be accessible by DFT. A small conductivity effective mass $m_{c}^{*}$ should lead to higher mobility, ${ }^{6}$ and have led to the reinvestigation of structures with very light bands such as SnTe. ${ }^{7}$ Valley degeneracy $\left(N_{\mathrm{v}}\right)$ has been shown to be critical for achieving high $z T^{8-12}$ Many of the best thermoelectric materials are known to have, recently have been found to have, or are being engineered to have high valley degeneracy, including: the lead and tin chalcogenides, $5,10,13$ diamond like copper selenides, ${ }^{14,15}$ Skutterudites, ${ }^{16} \mathrm{Mg}_{2} \mathrm{Si}^{11,}{ }^{17}$ Half Heuslers, ${ }^{18}$ and Zintl phases. ${ }^{19}$ While isolated pockets of Fermi surfaces can improve the quality factor, Fermi surface pockets connected by threads in the lead chalcogenides ${ }^{20}$ and the complex Fermi surfaces of bismuth telluride ${ }^{21}$ have also been suggested to be beneficial for $z T$.

Here, we show that DFT calculations can be used to compute two types of effective masses characteristic of the electronic structure independent of the scattering mechanism. An inertial mass $m_{c}^{*}$ from the electrical conductivity and a density of states (DOS) mass $m_{\mathrm{S}}^{*}$ from the Seebeck coefficient and carrier concentration. Then we introduce the ratio of these two masses as the Fermi Surface complexity factor $\left(N_{\mathrm{v}}^{*} K^{*}\right)=\left(m_{\mathrm{S}}^{*} / m_{\mathrm{c}}^{*}\right)^{3 / 2}$, which we recognize as related to valley degeneracy $N_{\mathrm{v}}$ and carrier pocket anisotropy $K$.

\section{RESULTS}

Boltztrap conductivity mass $\left(m_{c}^{*}\right)$

The conductivity effective mass is computed directly from the Boltztrap calculation for electrical conductivity, $\left(m_{c}^{*}\right)^{-1}=\sigma / n e^{2} \tau$ using the constant relaxation time approximation (CRTA). This definition has been used to conduct a high-throughput search for low hole and electron low effective mass (high mobility) transparent conductive oxides ${ }^{22-24}$ and also for analyzing specific thermoelectric materials. ${ }^{25,} 26$ Specifically using the notation of Madsen and $\operatorname{Singh}^{27}$ the effective mass tensor can be computed from

Eq. (12) of ref. 27

$\left(m_{c_{a \beta}}^{*}(T ; \mu)\right)^{-1}=\frac{\sigma_{\alpha \beta}(T ; \mu)}{e^{2} \tau} \times \frac{1}{n(T ; \mu)}$

Where $e$ is the electron charge and $\tau$ is the user-specified constant relaxation time. When multiple bands contribute to conduction, $m_{c}^{*}$ is a weighted average over all contributing bands.

The net charge carrier concentration $n$ (or doping concentration) is measured relative to a band edge and is a positive quantity whether electrons or holes are dominant charge carriers. In BoltzTraP, $N_{\text {val, }}$ the number of valence electrons/cell required to place the Fermi level in the band gap to make the material "undoped" at $0 \mathrm{~K}$ is set by the user. Thus $\frac{N_{\text {val }}}{V}=$ $\int g(\epsilon) f_{\mu=\text { undoped }}(T=0 K ; \epsilon) d \epsilon$ where $V$ is the volume of the unit cell $\left(\mathrm{cm}^{3}\right), g(\epsilon)$ is the density of states (states/volume/Energy), and $f_{\mu=\text { undoped }}(T ; \epsilon)$ is the Fermi-Dirac distribution function where the electron chemical potential $\left(\mu\right.$ or $E_{\mathrm{F}}$ ) is that of the undoped material. The doping concentration is then $n=\mid \frac{N_{\text {val }}}{V}-$ $\int g(\epsilon) f_{\mu}(T ; \epsilon) d \epsilon \mid$ using the notation of Madsen and Singh, ${ }^{27}$ which is directly computed from the $N$ in the BoltzTrap output as $n(T ; \mu)$ $=(|N(T ; \mu)|) /(V)$.

The conductivity mass tensor $\left(m_{c}^{*}\right)$ should be a good representation of the band structure relevant to electrical conductivity independent of the scattering mechanism. This is a more relevant output from Boltztrap than conductivity ( $\sigma$ ) as CRTA is typically a poor approximation for scattering in real thermoelectric materials. Also while $\sigma$ changes rapidly in a semiconductor with differing Fermi level (or $n$ ), $m_{c}^{*}$ should be constant to a firstorder approximation as demonstrated in Fig. 1 making it a more robust descriptor of the BoltzTraP calculation result. Like conductivity, the drift mobility $\mu_{\mathrm{d}}$ requires knowledge of the scattering through $\tau$. Thus, while mobility could be easily computed from BoltzTraP:

$\mu_{\mathrm{d}_{\alpha \beta}}(T ; \mu)=\frac{\sigma_{\alpha \beta}(T ; \mu)}{e} \times \frac{1}{n(T ; \mu)}$

the uncertainty of $\tau$ makes effective mass $m_{c}^{*}$, not mobility $\mu_{d}$, the parameter that most directly represents the electronic structure.

Note that the effective mass $m_{c}^{*}$ used here differs from the commonly used definition involving the second derivative of a particular band dispersion: $1 / m_{\alpha \beta}^{*}=\left(1 / \hbar^{2}\right)\left(\partial^{2} E / \partial k_{\alpha} \partial k_{\beta}\right)$ either evaluated at a particular k-point (energy) or evaluated at the band edge. In general, particularly for complex band structures, these varying definitions can give different values for effective mass. It is mathematically equivalent (by integration by parts ${ }^{28}$ ) to use the second derivative mass, but one must remember that this second derivative mass must be averaged over the entire band of filled states at $0 \mathrm{~K}$. Thus using the second derivative, the effective mass $m_{c_{\alpha \beta}}^{*}(T ; \mu)$ requires an integration over all states weighted by the Fermi function. ${ }^{23}$ While $m_{c}^{*}$ is a good measure of the average inertial effective mass of electrons in a system, it does not indicate much about the density of electronic states in a system that has multiple bands.

\section{Boltztrap Seebeck mass $\left(m_{\mathrm{s}}^{*}\right)$}

The effective density of electronic states can be estimated through the Seebeck coefficient characterized by the DOS effective mass: $m_{\mathrm{s}}^{*}{ }^{26}$ This can be performed in analogy to the Pisarenko plot ( $S$ vs $n$ ), which is commonly used to estimate an effective mass $m_{\mathrm{S}}^{*}$ from experimental data. ${ }^{29,} 30$ Treating the BoltzTraP transport results as if it were experimental data, we solve for an effective reduced chemical potential $\eta_{\text {eff }}$ that yields the computed Seebeck coefficient. For each BoltzTraP calculated pair of $S(T ; \mu)$ (using $1 / 3$ the trace of the $S_{i j}(T ; \mu)$ tensor of Eq 16 in Madsen and Singh ${ }^{27}$ ) and $n(T ; \mu)$ we solve for a $m_{S}^{*}(T ; \mu)$. First, from $S(T ; \mu)$ we find the reduced chemical potential $\eta_{\text {eff }}$ that would give that same Seebeck coefficient for a single parabolic band:

$S(T ; \mu)=\frac{k_{\mathrm{B}}}{q}\left[\frac{(2+\lambda)}{(1+\lambda)} \frac{F_{1+\lambda}\left(\eta_{\mathrm{eff}}\right)}{F_{\lambda}\left(\eta_{\mathrm{eff}}\right)}-\eta_{\mathrm{eff}}\right]$

Where $\lambda$ is the scattering exponent, $q$ is $-e$ for electrons and $+e$ for holes, and $F_{1+\lambda}$ are the Fermi functions given by:

$F_{j}(\eta)=\int_{0}^{\infty} \frac{\epsilon^{j} d \epsilon}{1+e^{\epsilon-\eta}}$

We adjust the common scattering assumption, which is usually by acoustic phonons in experiments, to coincide with the CRTA used in BoltzTraP (i.e., set $\lambda=1 / 2$ ). The reduced chemical potentials $\left(\eta_{\text {eff }}=\left(\mu-E_{\mathrm{B}}\right) /\left(k_{\mathrm{B}} T\right)\right)$ are relative to the band edge where $E_{\mathrm{B}}$ is the energy of the (valence or conduction) band edge and the sign of $\eta$ is always such that $\eta_{\text {eff }}>0$ has chemical potential ( $\mu$ in Madsen and Singh; ${ }^{27} E_{\mathrm{F}}$ in BoltzTraP output) in the band.

Then, using the effective reduced chemical potential $\eta_{\text {eff, we }}$ find the effective mass $m_{\mathrm{S}}^{*}(T ; \mu)$ that would give the $n(T ; \mu)$ calculated from Boltztrap.

$n(T ; \mu)=\frac{1}{2 \pi^{2}}\left(\frac{2 m_{\mathrm{S}}^{*}(T ; \mu) k_{\mathrm{B}} T}{\hbar^{2}}\right)^{\frac{3}{2}} F_{1 / 2}\left(\eta_{\mathrm{eff}}\right)$

It should be noted that $m_{S}^{*}$ is a scalar quantity and not a tensor unlike $m_{c_{\alpha \beta}}^{*}$. Although the Seebeck coefficient $S_{i j}(T ; \mu)$ is a tensor, for 

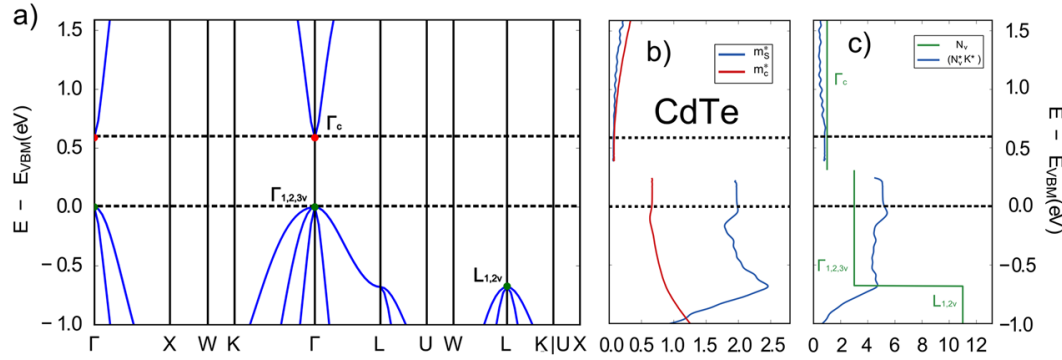

Wave Vector

$\mathrm{m} / \mathrm{m} 0$

$\mathrm{N}_{\mathrm{v}}$
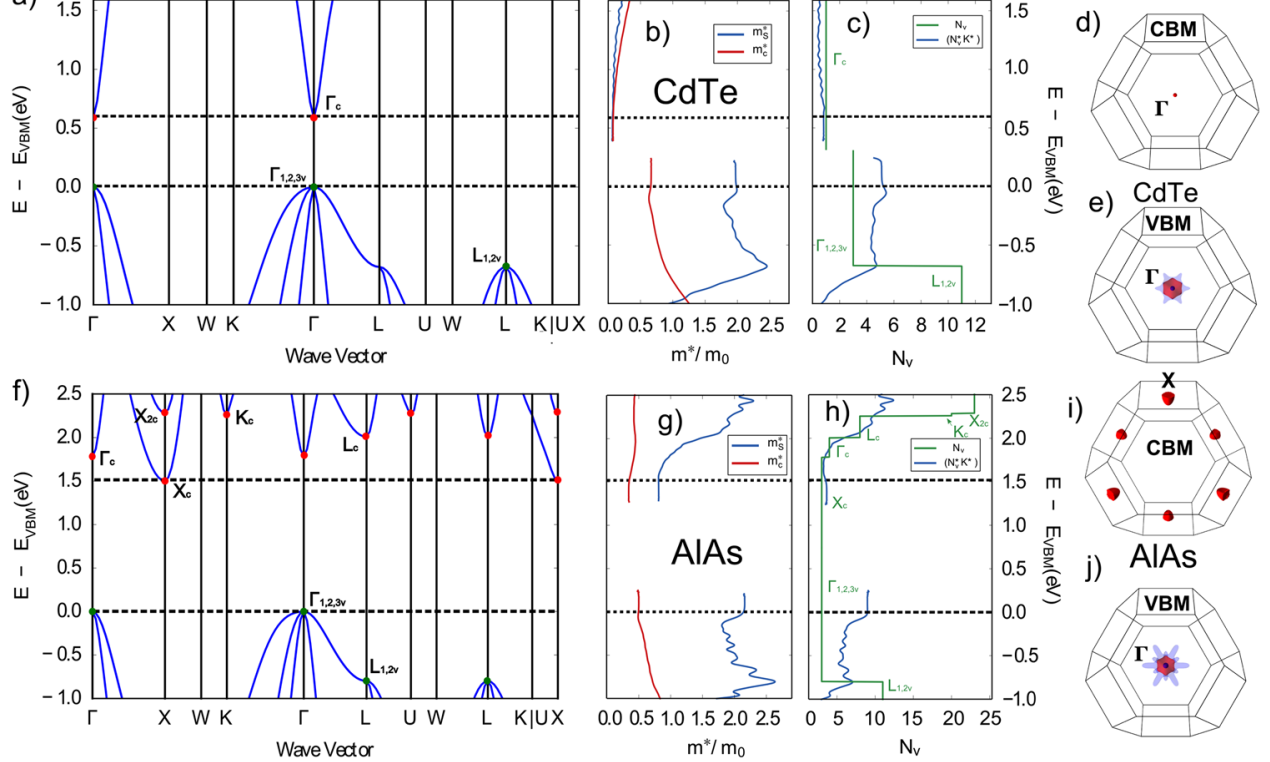

Fig. 1 Effective masses and complexity factor at $300 \mathrm{~K}$ from BoltzTraP for some relatively simple structures CdTe (a-e) and AlAs (f-j). $\mathbf{a}, \mathbf{f}$ Computed electronic band structure, $\mathbf{b}, \mathbf{g}$ Conductivity mass, $m_{\mathrm{c}}^{*}$, versus Fermi level and Seebeck DOS mass, $m_{\mathrm{S}}^{*}$, as functions of the Fermi level across the valence and conduction bands. $\mathbf{c}, \mathbf{h}$ Fermi surface complexity factor $N_{\mathrm{v}}^{*} K^{*}$ and true valley degeneracy $N_{\mathrm{v}}$. $\mathbf{d}$, i Primary conduction Fermi surface $(0.03 \mathrm{eV}$ above the conduction band edge (CBM) in CdTe and $0.1 \mathrm{eV}$ above the band edge for AIAs), and $\mathbf{e}$, $\mathbf{j}$ Valence band Fermi surface $(0.05 \mathrm{eV}$ below the valence band edge (VBM) for both CdTe and AIAs). The valence band Fermi surfaces are colored differently for the three degenerate bands

parabolic bands of the same sign, and for $\tau$ that may depend on energy but not direction, ${ }^{26}$ the Seebeck only depends on the reduced chemical potential $\eta_{\text {eff }}$ and the scattering exponent $\lambda$ both of which are scalars. Considering that the density of states $g(\epsilon)$, is also a scalar quantity it is appropriate that a density of states effective mass such as $m_{\mathrm{S}}^{*}$ should also be a scalar quantity. This single parabolic band Seebeck coefficient likely represents the thermopower from the configurational entropy of the electrons in the available states represented by $g(\epsilon)$, which would support our interpretation of $m_{\mathrm{S}}^{*}$ as an appropriate measure of the density of states effective mass.

The density of states effective mass is a convenient single metric to describe the density of states. Like $m_{c^{\prime}}^{*} m_{\mathrm{S}}^{*}$ remains relatively constant with doping while $S, \sigma, g, n$ change, and, therefore, $m_{\mathrm{S}}^{*}$ is a better descriptor of the density of states for semiconductors than any single value of $g$ (recall that $g(\epsilon)=$ $\left(8 \pi \sqrt{2} / h^{3}\right) m^{* 3 / 2} \sqrt{\epsilon}$ is a typical description for $g$ of a semiconductor). Even though there may be multiple or nonparabolic bands, a single effective mass is the best first order characterization of a semiconductor and is accurate within the uncertainty of measurements on new materials. Particularly for use in thermoelectrics, $m_{\mathrm{S}}^{*}$ is anticipated to be more relevant than an effective mass from a direct comparison to the calculated density of states $(g)^{2}$ because it most directly relates to the Seebeck coefficient both in theory and experiment.

Effective valley degeneracy $\left(N_{\mathrm{v}}^{*}\right)$

A Fermi surface consisting of $N_{v}$ identical, isolated surfaces will have total number of electronic states that is $N_{v}$ times the number of states in each isolated surface. If each isolated surface can be described with a density of states mass $m_{b}^{*}\left(\right.$ recall $\left.g(\epsilon) \propto m_{b}^{* 3 / 2}\right)$ for each band or pocket then the total density of states mass, here computed using the Seebeck coefficient, is $m_{\mathrm{s}}^{*}=N_{\mathrm{v}}^{2 / 3} m_{\mathrm{b}}^{*}$. Valley degeneracy then manifests itself by increasing the density of states effective mass relative to the single valley effective mass $\left(m_{\mathrm{b}}^{*}\right)$, which should be related to the inertial mass $m_{\mathrm{c}}^{*}$. Symmetry imposed valley degeneracy results from band extrema that exist at low symmetry points in a high symmetry crystal or orbital degeneracy of the constituent atoms. In order to maximize $N_{\mathrm{v}}$, the band extrema should be off high symmetry points (such as gamma), which is influenced by the symmetry of the most relevant atomic orbitals. ${ }^{31}$

When multiple bands contribute to conduction but they are not exactly degenerate (band extrema not at the same energy), we consider this an increased effective valley degeneracy. One can approximate $N_{v}^{*}$ by counting the total number of charge carrier Fermi surfaces (as in done for calculating $N_{\mathrm{v}}$ in Figs. 1 and 2, or within a particular energy window. ${ }^{2}$ We think of effective valley degeneracy, $N_{\mathrm{v}}^{*}$, as describing the effective number carrier pockets contributing to conduction, where the contribution may only be partial if that pocket is displaced by more than $\sim k_{\mathrm{B}} T$ from the chemical potential $(\mu)$.

\section{Effective anisotropy factor $\left(K^{*}\right)$}

Only in the simplest cases can Fermi surfaces can be described as spherical pockets; many materials contain more complicated Fermi surfaces. The next level of complexity involves ellipsoidally shaped pockets where the anisotropy parameter, $K=m_{\|}^{*} / m_{\perp}^{*}$, quantifies the degree of anisotropy. ${ }^{32}$ Many material systems have been shown to display $K$ different from unity including: $\mathrm{Si} / \mathrm{Ge}^{33,}, 34$ IV-VI materials, ${ }^{32,} 35,36$ III-V materials, ${ }^{37}$ and others. ${ }^{38}$ The conductivity effective mass of such systems is calculated from the harmonic average along each direction: $m_{c}^{*}=3\left(m_{1}^{*-1}+2 m_{\perp}^{*-1}\right)$, which determines the carrier mobility $\left(\mu=e \tau / m_{c}^{*}\right)$. The conductivity mass $m_{c}^{*}$ is, in general, different from the single valley density of states mass $m_{b}^{*}$ (geometric average: $\left.m_{\mathrm{b}}^{*}=\left(m_{\|}^{*} m_{\perp}^{* 2}\right)^{1 / 3}\right)$; they are equal only for spherical pockets $\left(K=m_{\|}^{*} / m_{\perp}^{*}=1\right)$. For non-ellipsoidally shaped Fermi surfaces, we can define the effective anisotropy parameter $K^{*}$ in terms of the effective masses: ${ }^{26}$

$$
K^{*}=\left(\frac{m_{\mathrm{b}}^{*}}{m_{\mathrm{c}}^{*}}\right)^{3 / 2}=\frac{(2 K+1)^{3 / 2}}{3^{3 / 2} K}
$$



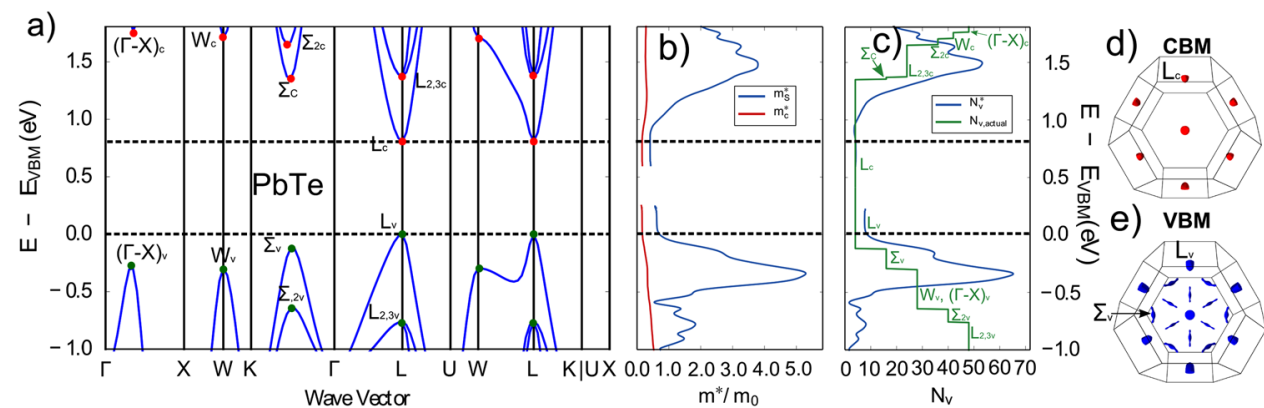

e) vBM
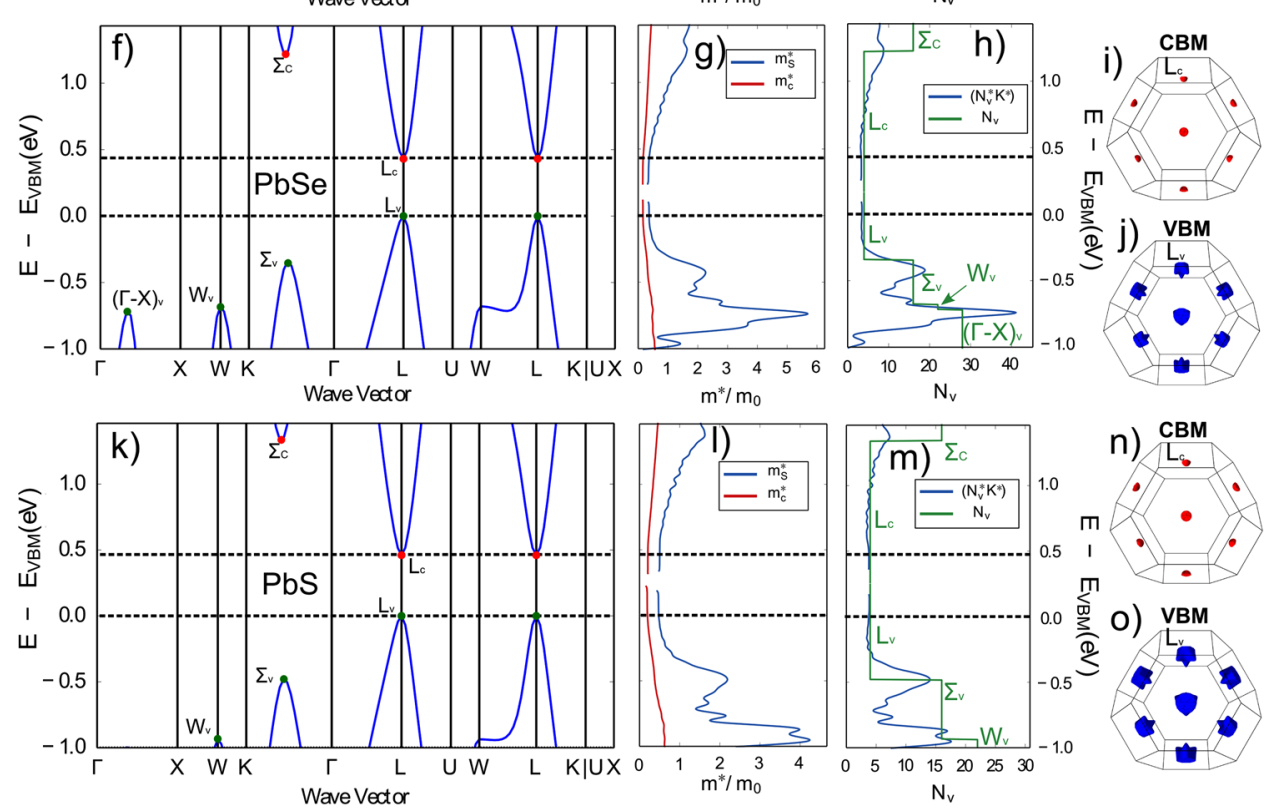

n) $\mathrm{CBM}$
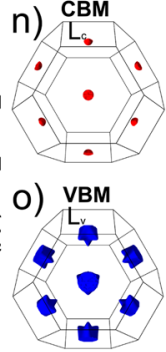

Fig. 2 Band structure, $m_{\mathrm{c}}^{*}$ and $m_{\mathrm{S}}^{*}$ versus Fermi level, and $N_{\mathrm{v}}^{*} K^{*}$ at $300 \mathrm{~K}$ versus Fermi level, and valence and conduction band Fermi surfaces for $\mathrm{PbTe}(\mathbf{a}-\mathbf{e}), \mathrm{PbSe}(\mathbf{f}-\mathbf{j})$, and $\mathrm{PbS}(\mathbf{k}-\mathbf{o})$. The valence and conduction band edges are shown as a dashed line. Fermi surfaces are drawn at $0.1 \mathrm{eV}$ above the conduction band edge (CBM) for PbTe, $\mathrm{PbSe}$, and $\mathrm{PbTe}$, respectively, or $0.13,0.3$, and $0.4 \mathrm{eV}$ below the valence band edge (VBM) for $\mathrm{PbTe}, \mathrm{PbSe}$, and $\mathrm{PbS}$, respectively

Fermi surface complexity factor $\left(N_{\mathrm{v}}^{*} K^{*}\right)$

Combining the effects of valley degeneracy $N_{v}^{*}$ and anisotropy $K^{*}$ we define the Fermi surface complexity factor, $\left(N_{\mathrm{v}}^{*} K^{*}\right)$ in terms of the effective masses $m_{\mathrm{c}}^{*}$ and $m_{\mathrm{S}}^{*}$ we derive from DFT calculations:

$\left(N_{\mathrm{v}}^{*} K^{*}\right)=\left(\frac{m_{\mathrm{s}}^{*}}{m_{\mathrm{c}}^{*}}\right)^{3 / 2}$

Although it is not clear how to define the single valley effective mass $\left(m_{\mathrm{b}}^{*}\right)$ in general, for simple cases of multiple, degenerate, ellipsoidal Fermi surface pockets we concluded $N_{\mathrm{v}}^{*}=\left(m_{\mathrm{s}}^{*} / m_{\mathrm{b}}^{*}\right)^{3 / 2}$ and $K^{*}=\left(m_{\mathrm{b}}^{*} / m_{\mathrm{c}}^{*}\right)^{3 / 2}$. So while we do not to calculate or even clearly define $N_{\mathrm{v}}^{*}$ or $K^{*}$ individually, we can clearly define $\left(N_{\mathrm{v}}^{*} K^{*}\right)$ through the equation above. Because $m_{c_{\beta}}^{*}$ is, in general, a tensor while $m_{S}^{*}$ is a scalar, $\left(N_{v}^{*} K^{*}\right)_{\alpha \beta}$ is most generally a second rank tensor like $m_{c_{\alpha \beta}}^{*}$. For simplicity in this work, only isotropic compounds are demonstrated below so that only scalar values are needed.

The Fermi surface complexity factor $N_{\mathrm{v}}^{*} K^{*}$ can be a good indicator of the effective valley degeneracy $N_{\mathrm{v}}^{*}$. Compared to the valley degeneracy $N_{v}^{*}$, which can easily be three or more, the anisotropy factor $K^{*}$, in some cases, may only lead to small deviations from unity, resulting in a complexity factor $N_{\mathrm{v}}^{*} K^{*} \approx N_{\mathrm{v}}^{*}$. For ellipsoidal Fermi pockets a factor of two difference in the directional effective mass, $m_{\|}^{*} / m_{\perp}^{*}=2$, results only in $K^{*}=1.08$ while a highly anisotropic $m_{\|}^{*} / m_{\perp}^{*}=12$ is required to get just $K^{*}=2$.
The Fermi surface complexity factor $N_{\mathrm{v}}^{*} K^{*}$ may also be a good indicator of the suitability of a material for thermoelectrics as noted by Parker et al. ${ }^{26}$ who also considered a ratio similar to $m_{\mathrm{s}}^{*} / m_{\mathrm{c}}^{*}$. Complex Fermi surfaces have been shown to be beneficial for $z T$. Complexity in the form of high valley degeneracy $N_{\mathrm{v}}$ (multiple carrier pockets in the Fermi surface contributing to conduction) such as found in $\mathrm{PbTe}^{39}$ is widely recognized to be beneficial to thermoelectric performance as seen in the quality factor. However, other complexities such as narrow threads of Fermi surface has also been identified to improve thermoelectric transport. $^{20,36,40,41}$

The advantage of anisotropy factor $K^{*}$ may be most apparent when the scattering is also complex. Separating the scattering time $\tau_{0}$ from the quality factor ${ }^{42}$ ] results in a quality factor strongly dependent on $N_{\mathrm{v}}^{*} K^{*}$ :

$B=\frac{k_{\mathrm{B}}^{2} T\left(k_{\mathrm{B}} T\right)^{3 / 2}}{3 \pi^{2} \hbar^{3}} \frac{\tau_{0}}{K_{\mathrm{L}}} N_{\mathrm{v}}^{*} K^{*}\left(m_{\mathrm{c}}^{*}\right)^{1 / 2}$

In these cases where the Fermi surface has additional complexity beneficial to thermoelectrics, $N_{\mathrm{v}}^{*} K^{*}$ may be an auspicious metric for thermoelectric materials.

\section{DISCUSSION}

II-VI and III-V Materials-CdTe and AIAs

As a first example, the conduction band of $\mathrm{CdTe}$ is centered directly at $\Gamma\left(N_{v}=1\right)$ and has a spherical Fermi surface (as shown in 
Fig. 1d). This simple band structure shows the effective masses $\left(m_{\mathrm{S}}^{*}\right.$ and $m_{\mathrm{c}}^{*}$ ) as computed from calculated Boltztrap data (shown in Fig. 1 as described earlier) are constant with varying doping (Fermi level) and roughly consistent with experimental and traditionally calculated values of $m^{*} .43$ The Fermi surface complexity factor $\left(N_{\mathrm{v}}^{*} K^{*}\right)$ also shown in Fig. $1 \mathrm{c}$ is very close to 1 for the conduction band, consistent with a single $\left(N_{v}^{*}=1\right)$ spherical pocket $\left(K^{*}=1\right)$. The computed $N_{\mathrm{v}}^{*} K^{*}$ is approximately 1 over a wide range of Fermi levels even as the Fermi level is moved deeper into the $\Gamma$ band. A slight decrease in value and increase in noise is observed as the Fermi level moves farther into the band presumably due to sensitivity of $m_{\mathrm{S}}^{*}$ to the small variations in Seebeck at these Fermi levels.

Next, consider the slightly more complicated conduction band of AIAs, which has multiple carrier pockets at different points in the Brillouin zone (Fig. 1i), as the Fermi level is moved into the conduction band. The AlAs Fermi surface for the primary conduction band at $X$ has multiple pockets $\left(N_{v}=3\right)$ (Fig. 1i). We calculate $\left(N_{\mathrm{v}}^{*} K^{*}\right)=3.5$ near the conduction band edge consistent with our expectation of $N_{\mathrm{v}} \sim 3$ and $K^{*}$ close to 1 .

One might expect that $N_{v}^{*}$ should make a stepwise transition from one value of $N_{\mathrm{v}}$ to another as the Fermi level approaches and enters the additional band. This behavior is observed in Fig. $1 \mathrm{~h}$ where $N_{\mathrm{v}}^{*} K^{*}$ is compared to a hypothetical $N_{\mathrm{v}}$ just by considering the symmetry of the extrema alone (green line in Fig. $1 \mathrm{~g}, N_{\mathrm{v}}\left(E_{\mathrm{F}}\right)=$ $\sum N_{\mathrm{v}, i} \mathrm{H}\left(E_{\mathrm{F}}-E_{\mathrm{i}}\right)$ where $\mathrm{H}$ is the Heaviside step function and $E_{i}$ is the energy of the $i^{\text {th }}$ band extrema). ${ }^{2}$ As the Fermi level moves into the conduction band, we reach the $\Gamma_{\mathrm{c}}\left(N_{\mathrm{v}}=1,0.28 \mathrm{eV}\right.$ above $\left.X_{\mathrm{c}}\right)$ and $L_{\mathrm{c}}\left(N_{\mathrm{v}}=4,0.51 \mathrm{eV}\right.$ above $\left.X_{\mathrm{c}}\right)$ bands where the total $N_{\mathrm{v}}$ increases to 4 and 8 , respectively. The Fermi surface complexity factor $\left(N_{v}^{*} K^{*}\right)$ increases steadily from the band edge resulting in a value of 3.5 and 6.1 at the $\Gamma_{c}$ and $L_{c}$ band edge energies, respectively. The thermoelectric Fermi surface complexity factor $\left(N_{\mathrm{v}}^{*} K^{*}\right)$ mirrors the true $N_{\mathrm{v}}$ both qualitatively and quantitativelyconsistent with an anisotropy component, $K^{*}$, that is not far from unity. The Supplementary material includes $m_{\mathrm{s}}^{*}, m_{\mathrm{c}}^{*}, N_{\mathrm{v}},\left(N_{\mathrm{v}}^{*} K^{*}\right)$ for the other III-V materials (Table S1).

Enhancement in $K^{*}$ appears in the valence band of both CdTe and AIAs, which consist of three degenerate bands: $\Gamma_{1,2,3 v}$, with different effective masses (light hole, heavy hole, and split-off band) due to $p$-orbital degeneracy. ${ }^{31}$ As a result, the Fermi surface - even though it is centered at the $\Gamma$ point-will have a non-trivial topology (as described by Mecholsky et al. ${ }^{41}$ for silicon), which appears to result in a larger $K^{*}$ component to the Fermi surface complexity factor and a $N_{\mathrm{v}}^{*} K^{*}$ that exceeds the expected degeneracy of $N_{v}=3$ for $\Gamma_{1,2,3 v}$. For the valence band, $\left(N_{v}^{*} K^{*}\right)=6$ and 9 for CdTe and AlAs, respectively. Mecholsky et al. ${ }^{41^{v}}$ shows that warped, non-ellipsoidal Fermi surfaces, which result from the combination of light and heavy bands, significantly influence the electronic transport in these systems altering the equivalent effective masses.

\section{IV-VI Materials-PbS-PbSe-PbTe}

The lead chalcogenides (including $\mathrm{PbTe}, \mathrm{PbSe}^{42,}{ }^{44} \mathrm{PbS}^{45}$ and their alloys ${ }^{46-54}$ ) are known to be good thermoelectric materials partly because of their complex electronic structure. The Fermi surface complexity factor and effective masses were also computed for these IV-VI compounds. For PbS and PbSe, the conduction band at the L-point shows significant valley degeneracy of $N_{\mathrm{v}}=4$ shows $N_{\mathrm{v}}^{*} K^{*}=4$ as expected for chemical potential near the band edge. The primary valence band, also with $N_{\mathrm{v}}=4$ has a nearby secondary valence band along the $\Sigma$ line, with its own $N_{\mathrm{v}}=12$, which can be seen by a rapidly rising $N_{\mathrm{v}}^{*} K^{*}$ that approaches the simple sum of these valley degeneracies.

An exceptionally high $N_{v}^{*} K^{*}$ is also found in p-type $\mathrm{PbTe}$, above that expected from $N_{v}^{*}$ of $L$ and $\Sigma$ bands indicating a significant contribution from $K^{*}$ or a new band. In p-type PbTe the complex

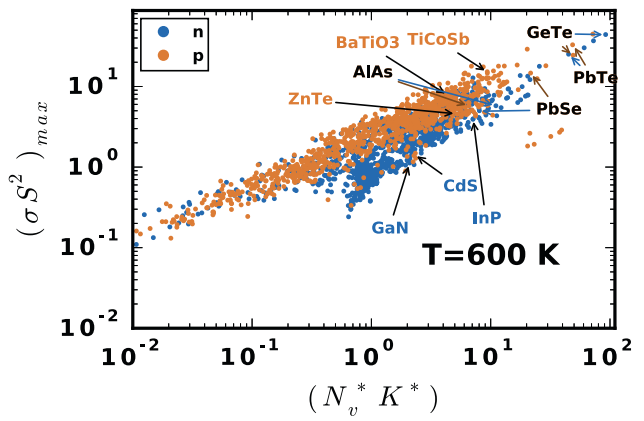

Fig. 3 Maximum power factor for 2300 cubic compounds plotted as a function of the Fermi surface complexity factor (evalulated at the Fermi level which yields the maximum power factor) at $T=600 \mathrm{~K}$

Fermi surface characterized by threads which develop between the $L_{-v}$ and $\Sigma_{-v}$ pockets, which have been concluded to contribute to the high thermoelectric performance. ${ }^{8,55-59}$ This could be due to the large surface area to volume ratio of the thread-like states, which leads to an inherently large mobility and quality factor (and corresponding large $K^{*}$ ). Compared to $\mathrm{PbS}$ and $\mathrm{PbSe}$, the bands in $\mathrm{PbTe}$ are closer in energy (e.g., the $L$ and $\Sigma$ bands are computed to be only $\sim 0.12 \mathrm{eV}$ apart) and these energies may change with temperature. For example, in $\mathrm{PbTe}$, the $\mathrm{L}$ and $\Sigma$ bands are thought to shift with temperature, eventually converging at $\sim 700 \mathrm{~K}$ (ref. 56 ).

\section{High-throughput computation}

The vast electronic structure database constructed through the Materials Project allows for large-scale screening of semiconductors for thermoelectrics, transparent conductors as well as other applications. By combining DFT and BoltZtraP (using the CRTA) thousands of compounds can be screened for effective mass $\left(m_{\mathrm{c}}^{*}, m_{\mathrm{S}}^{*}\right)$ and complexity factor $N_{\mathrm{v}}^{*} K^{*}$ (Supplementary Table I).

Figure 3 shows the correlation between $\left(N_{\mathrm{v}}^{*} K^{*}\right)$ and the calculated maximum (Fermi level-dependent) power factor (assuming constant $\tau=10^{-14} \mathrm{~s}$ ) for the large group of compounds ( 2300 isotropic compounds) at $600 \mathrm{~K}$. We can see a good correlation between the calculated Fermi surface complexity factor and the maximum attainable power factor; this is expected since the quality factor for constant relaxation time is expected to scale according to Eqn. 9. Data regarding the maximum power factor and $N_{\mathrm{v}}^{*} K^{*}$ is included in the Supplementary material.

While experimental conductivity values are difficult to reproduce within the constant relaxation time, ${ }^{1}$ it is remarkable that known thermoelectric materials such as PbTe, GeTe, TiCoSb show up with a high constant relaxation time power factor and a high Fermi surface complexity factor. This indicates that, at least for screening and ranking, the Fermi surface complexity factor is an effective descriptor.

\section{CONCLUSIONS}

Both a density of states effective mass, $m_{\mathrm{s}}^{*}$ and inertial effective mass $m_{c}^{*}$ can be extracted even from complex DFT band structures using the result of $\sigma$ and $S$ transport calculations, such as done in BoltzTraP, even if the scattering mechanism is not known. Because $m_{\mathrm{c}}^{*}$ and $m_{\mathrm{S}}^{*}$ are less influenced by $\tau$ and scattering mechanism than $\sigma$ and $S$, they are better descriptors of a band structure's contribution to transport than a CRTA value of $\sigma$ and $S$ itself. We interpret the ratio of these two masses as a Fermi Surface Complexity Factor $\left(N_{\mathrm{v}}^{*} K^{*}\right)$, which should be influenced by effective valley degeneracy $N_{v}^{*}$ and anisotropy $K^{*}$, both beneficial to thermoelectric performance.

We have analyzed the maximum thermoelectric power factors and for a large set compounds from the Materials Project to show 
that $\left(N_{\mathrm{v}}^{*} K^{*}\right)$ appears to correlate well with thermoelectric performance. A larger than expected $\left(N_{\mathrm{v}}^{*} K^{*}\right)$ is found in the lead chalcogenide semiconductors, is likely as a result of non-trivial topological features of the Fermi surface. Combining highthroughput DFT with Boltztrap calculations to extract commonly understood quantities such as effective mass from band structures have the potential to impact many applications. By predicting how these properties evolve with doping and chemistry will enable future band engineering for even broader use.

\section{METHODS}

$\mathrm{Ab}$ initio computations in this section are from the Materials Project database ${ }^{60}$ and use DFT within the generalized gradient approximation (GGA) or GGA $+U$ in some compounds ${ }^{1}$ in the Perdew-Burke-Ernzheroff formulation. ${ }^{61}$ Calculations were performed using the VASP software and projector augmented-wave pseudopotentials. ${ }^{62}$ Bolztrap calculations were computed using the open-source code ${ }^{27}$ along with analysis and plotting software from pymatgen. ${ }^{63}$

High-throughput calculations of the Fermi surface complexity factor are analyzed in Fig. 3, but we limit the analysis to isotropic compounds (maximum deviation in the eigenvalues of the power factor tensor of $<3 \%$ along any direction) and those with a maximum optimum carrier concentration $<1 \times 10^{21} \mathrm{~cm}^{-3}$. We also chose to remove compounds that were not particularly stable or those that were metallic (we required that the energy above the convex hull be $<0.05 \mathrm{eV} /$ atom, and that the band gap be $>0.03 \mathrm{eV}$ ). The entire Fermi Surface Complexity Factor results for calculations at $600 \mathrm{~K}$ (with unstable compounds removed) is included in the Supplementary material under the conditions: $1 \times 10^{20} \mathrm{~cm}^{-3}$, maximum power factor conditions, and maximum $z T$ conditions (assuming $\tau=1 \times$ $10^{-14} s$ and $\left.\kappa_{\mathrm{L}}=0.5(\mathrm{~W}) /(m-K)\right)$.

The band structures we used do not include spin-orbit coupling effects. However, our approach to compute effective masses and Fermi surface complexity could easily be applied on data generated with spin-orbit coupling.

Additional calculation details specific to the III-V or IV-VI materials are included in the Supplementary material.

\section{ACKNOWLEDGEMENTS}

We thank Georg Madsen, Eric Toberer, Vladan Stevanovic and David Singh for helpful discussions. Z.M.G. acknowledges contributions to the code base by Meera Kumar, a Caltech summer undergraduate researcher. This work was intellectually led by the Materials Project which is supported by the Department of Energy Basic Energy Sciences program under Grant No. EDCBEE, DOE Contract DE-AC02-05CH11231. This research used resources of the National Energy Research Scientific Computing Center, a DOE Office of Science User Facility supported by the Office of Science of the U.S. Department of Energy. The work was supported by the F.R.S.-FNRS project HTBaSE (contract no. PDR-T.1071.15). Computational resources were provided by the supercomputing facilities of the Université catholique de Louvain (CISM/UCL), the Consortium des Equipements de Calcul Intensif en Federation Wallonie Bruxelles de (CECI) funded by the F.R.S.-FNRS.

\section{AUTHOR CONTRIBUTIONS}

This strategy was conceived by G.J.S. and Z.M.G. The code to implement the calculations was written by Z.M.G. with input from G.L., H.Z., F.R., A.J., and G.H. The high-throughput calculations were managed by A.J., G.H., F.R., G.C. and K.P. The manuscript was written by Z.M.G., G.J.S., F.R., G.H. and A.J. and approved by all authors.

\section{COMPETING INTERESTS}

The authors declare no competing interest.

\section{REFERENCES}

1. Chen, W. et al. Understanding thermoelectric properties from high-throughput calculations: trends, insights, and comparisons with experiment. J. Mater. Chem. C. 4, 4414-4426 (2016).

2. Yan, J. et al. Material descriptors for predicting thermoelectric performance. Energ. Environ. Sci. 8, 983-994 (2014).
3. Yang, J. et al. Evaluation of half-heusler compounds as thermoelectric materials based on the calculated electrical transport properties. Adv. Funct. Mater. 18, 2880-2888 (2008).

4. Yang, J. et al. On the tuning of electrical and thermal transport in thermoelectrics: an integrated theory-experiment perspective. Npj Comput. Mater. 2, 15015 (2016).

5. Pei, Y., Wang, H. \& Snyder, G. J. Band engineering of thermoelectric materials. Adv. Mater. 24, 6125-6135 (2012).

6. Pei, Y., LaLonde, A. D., Wang, H. \& Snyder, G. J. Low effective mass leading to high thermoelectric performance. Energ. Environ. Sci. 5, 7963-7969 (2012).

7. Zhou, M. et al. Optimization of thermoelectric efficiency in SnTe: the case for the light band. Phys. Chem. Chem. Phys. 16, 20741-20748 (2014).

8. Pei, Y. Z. et al. Convergence of electronic bands for high performance bulk thermoelectrics. Nature. 473, 66-69 (2011).

9. Zhao, L. D., Dravid, V. P. \& Kanatzidis, M. G. The panoscopic approach to high performance thermoelectrics. Energ. Environ. Sci. 7, 251-268 (2014).

10. Tan, G. et al. Extraordinary role of $\mathrm{Hg}$ in enhancing the thermoelectric performance of p-type SnTe. Energ. Environ. Sci. 8, 267-277 (2015).

11. Liu, W. et al. Convergence of conduction bands as a means of enhancing thermoelectric performance of n-Type Mg2Si1-xSnx solid solutions. Phys. Rev. Lett. 108, 166601 (2012).

12. Tan, G. et al. High thermoelectric performance of $\mathrm{p}$-Type SnTe via a synergistic band engineering and nanostructuring approach. J. Am. Chem. Soc. 136, 7006-7017 (2014).

13. Dong, X., Yu, H., Li, W., Pei, Y. \& Chen, Y. First-principles study on band structures and electrical transports of doped-SnTe. J. Materiomics 2, 158-164 (2016).

14. Zhang, J. et al. High-performance pseudocubic thermoelectric materials from non-cubic chalcopyrite compounds. Adv. Mater. 26, 3848-3853 (2014).

15. Zeier, W. G. et al. Band convergence in the non-cubic chalcopyrite compounds Cu2MGeSe4. J. Mater. Chem. C. 2, 10189-10194 (2014).

16. Tang, Y. et al. Convergence of multi-valley bands as the electronic origin of high thermoelectric performance in CoSb3 skutterudites. Nat. Mater. 14, 1223-1228 (2015).

17. Zaitsev, V. K. et al. Highly effective Mg2Si1-xSnx thermoelectrics. Phys. Rev. B. 74, 045207 (2006).

18. Fu, C. et al. High band degeneracy contributes to high thermoelectric performance in p-type half-heusler compounds. Adv. Energy Mater. 4, (2014).

19. Zhang, J. et al. Designing high-performance layered thermoelectric materials through orbital engineering. Nat. Commun. 7, (2016).

20. Parker, D., Chen, X. \& Singh, D. J. High three-dimensional thermoelectric performance from low-dimensional bands. Phys. Rev. Lett. 110, 146601 (2013).

21. Shi, H., Parker, D., Du, M.-H. \& Singh, D. J. Connecting thermoelectric performance and topological-insulator behavior: $\mathrm{Bi} 2 \mathrm{Te} 3$ and $\mathrm{Bi} 2 \mathrm{Te} 2 \mathrm{Se}$ from first principles. Phys. Rev. Appl. 3, 014004 (2015).

22. Hautier, G., Miglio, A., Ceder, G., Rignanese, G.-M. \& Gonze, X. Identification and design principles of low hole effective mass p-type transparent conducting oxides. Nat. Commun. 4, 2292 (2013).

23. Hautier, G., Miglio, A., Waroquiers, D., Rignanese, G.-M. \& Gonze, X. How does chemistry influence electron effective mass in oxides? A high-throughput computational analysis. Chem. Mater. 26, 5447-5458 (2014).

24. Bhatia, A. et al. High-mobility bismuth-based transparent p-type oxide from highthroughput material screening. Chem. Mater. 28, 30-34 (2016).

25. Lykke, L., Iversen, B. B. \& Madsen, G. K. H. Electronic structure and transport in the low-temperature thermoelectric $\mathrm{CsBi}_{4} \mathrm{Te}_{6}$ : Semiclassical transport equations. Phys. Rev. B. 73, 195121 (2006).

26. Parker, D. S., May, A. F. \& Singh, D. J. Benefits of carrier-pocket anisotropy to thermoelectric performance: the case of p-type AgBiSe2. Phys. Rev. Appl. 3, 064003 (2015).

27. Madsen, G. K. H. \& Singh, D. J. BoltzTraP. A code for calculating band-structure dependent quantities. Comput. Phys. Commun. 175, 67-71 (2006).

28. Ashcroft, N. W. \& Mermin, N. D. Solid State Physics. (Holt, Rinehart, and Winston, 1976).

29. May, A. F. \& Snyder, G. J. in CRC Handbook of Thermoelectrics (ed. Rowe, D. M.) (CRC Press, 2012).

30. May, A. F., Toberer, E. S., Saramat, A. \& Snyder, G. J. Characterization and analysis of thermoelectric transport in n-type Ba8Ga16-xGe30+x. Phys. Rev. B. 80, 125205 (2009).

31. Zeier, W. G. et al. Thinking like a chemist: intuition in thermoelectric materials. Angew. Chem. Int. Ed. 55, 6826-6841 (2016).

32. Ravich, Y. I., Efimova, B. A. \& Smirnov, I. A. Semiconducting Lead Chalcogenides Vol. 5 (Plenum Press, 1970).

33. Dexter, R. N., Zeiger, H. J. \& Lax, B. Cyclotron resonance experiments in silicon and germanium. Phys. Rev. 104, 637-644 (1956).

34. Dresselhaus, G., Kip, A. F. \& Kittel, C. Cyclotron resonance of electrons and holes in silicon and germanium crystals. Phys. Rev. 98, 368-384 (1955). 
35. Allgaier, R. S. Magnetoresistance in PbS, PbSe, and PbTe at 295, 77.4, and 4.2K. Phys. Rev. 112, 828-836 (1958).

36. Chen, X., Parker, D. \& Singh, D. J. Importance of non-parabolic band effects in the thermoelectric properties of semiconductors. Sci. Rep 3, 3168 (2013).

37. Vurgaftman, I., Meyer, J. R. \& Ram-Mohan, L. R. Band parameters for III-V compound semiconductors and their alloys. J. Appl. Phys. 89, 5815-5875 (2001).

38. Lerner, L. S. Shubnikov-de Haas Effect in Bismuth. Phys. Rev. 127, 1480-1492 (1962).

39. LaLonde, A. D., Pei, Y., Wang, H. \& Jeffrey Snyder, G. Lead telluride alloy thermoelectrics. Mater. Today 14, 526-532 (2011).

40. Svane, A. et al. Quasiparticle self-consistent GW calculations for PbS, PbSe, and PbTe: Band structure and pressure coefficients. Phys. Rev. B. 81, 45120:245110-245121 (2010).

41. Mecholsky, N. A., Resca, L., Pegg, I. L. \& Fornari, M. Theory of band warping and its effects on thermoelectronic transport properties. Phys. Rev. B. 89, 155131 (2014)

42. Wang, H., Pei, Y., LaLonde, A. D. \& Snyder, G. J. Weak electron-phonon coupling contributing to high thermoelectric performance in n-type PbSe. Proc. Natl Acad. Sci. 109, 9705-9709 (2012).

43. Karazhanov, S. Z. \& Lew Yan Voon, L. C. Ab initio studies of the band parameters of III-V and II-VI zinc-blende semiconductors. Semiconductors. 39, 161-173 (2005).

44. Wang, H., Pei, Y. Z., LaLonde, A. D. \& Snyder, G. J. Heavily doped p-Type PbSe with high thermoelectric performance: an alternative for PbTe. Adv. Mater. 23, 1366-1370 (2011).

45. Kanatzidis, M. G. et al. Nanostructures boost the thermoelectric performance of PbS. J. Am. Chem. Soc. 133, 3460-3470 (2011).

46. Wang, H., Wang, J. L., Cao, X. L. \& Snyder, G. J. Thermoelectric alloys between $\mathrm{PbSe}$ and $\mathrm{PbS}$ with effective thermal conductivity reduction and high figure of merit. J. Mater. Chem. A 2, 3169-3174 (2014).

47. Zhao, L. D. et al. Raising the thermoelectric performance of $\mathrm{p}$-Type PbS with endotaxial nanostructuring and valence-band offset engineering using $\mathrm{CdS}$ and ZnS. J. Am. Chem. Soc. 134, 16327-16336 (2012).

48. Korkosz, R. J. et al. High ZT in p-Type (PbTe)1-2x(PbSe)x(PbS)x thermoelectric materials. J. Am. Chem. Soc. 136, 3225-3237 (2014).

49. Yamini, S. A. et al. Chemical composition tuning in quaternary p-type Pb-chalcogenides-a promising strategy for enhanced thermoelectric performance. Phys. Chem. Chem. Phys. 16, 1835-1840 (2014).

50. Lee, Y. et al. High-performance tellurium-free thermoelectrics: all-scale hierarchical structuring of p-Type PbSe-MSe Systems ( $\mathrm{M}=\mathrm{Ca}, \mathrm{Sr}, \mathrm{Ba}) . \mathrm{J}$. Am. Chem Soc. 135, 5152-5160 (2013).
51. Jaworski, C. M. et al. Valence-band structure of highly efficient p-type thermoelectric PbTe-PbS alloys. Phys. Rev. B 87, 045201-045210 (2013).

52. Zhang, Q. et al. Heavy doping and band engineering by potassium to improve the thermoelectric figure of merit in p-Type PbTe, PbSe, and PbTe1-ySey. J. Am. Chem. Soc. 134, 10031-10038 (2012).

53. Wang, H., Gibbs, Z. M., Takagiwa, Y. \& Snyder, G. J. Tuning bands of PbSe for better thermoelectric efficiency. Energ. Environ. Sci. 7, 804-811 (2014).

54. Kanatzidis, M. et al. Thermoelectrics from abundant chemical elements: highperformance nanostructured PbSe-PbS. J. Am. Chem. Soc. 133, 10920-10927 (2011).

55. Kim, H. \& Kaviany, M. Effect of thermal disorder on high figure of merit in $\mathrm{PbTe}$ Phys. Rev. B 86, 045210-045211 (2012).

56. Gibbs, Z. M. et al. Temperature dependent band gap in $\mathrm{PbX}(\mathrm{X}=\mathrm{S}, \mathrm{Se}, \mathrm{Te})$. Appl. Phys. Lett. 103, 262109 (2013).

57. Allgaier, R. S. \& Houston, B. B. Hall coefficient behavior and 2 nd valence band in lead telluride. J. Appl. Phys. 37, 302-309 (1966).

58. Allgaier, R. S. Valence bands in lead telluride. J. Appl. Phys. 32, 2185-2189 (1961).

59. Kolomoets, N. V., Vinogradova, M. N. \& Sysoeva, L. M. Valence band of PbTe. Sov. Phys. Semicond. 1, 1020-1024 (1968).

60. Jain, A. et al. A high-throughput infrastructure for density functional theory calculations. Comp. Mater. Sci. 50, 2295-2310 (2011).

61. Perdew, J. P., Burke, K. \& Ernzerhof, M. Generalized gradient approximation made simple. Phys. Rev. Lett. 77, 3865-3868 (1996).

62. Kresse, G. \& Furthmüller, J. Efficiency of ab-initio total energy calculations for metals and semiconductors using a plane-wave basis set. Comp. Mater. Sci. 6 15-50 (1996).

63. Ong, S. P. et al. Python materials genomics (pymatgen): a robust, open-source python library for materials analysis. Comp. Mater. Sci. 68, 314-319 (2013).

\section{(c) (i)}

This work is licensed under a Creative Commons Attribution 4.0 International License. The images or other third party material in this article are included in the article's Creative Commons license, unless indicated otherwise in the credit line; if the material is not included under the Creative Commons license, users will need to obtain permission from the license holder to reproduce the material. To view a copy of this license, visit http://creativecommons.org/licenses/by/ $4.0 /$

(c) The Author(s) 2017

Supplementary Information accompanies the paper on the npj Computational Materials website (doi:10.1038/s41524-017-0013-3). 